\title{
Establishment of the cell line, HeLa-CD14, transfected with the human CD14 gene
}

\author{
BO-TAO NING and YONG-MIN TANG \\ Division of Hematology-Oncology, The Children's Hospital of Zhejiang University School of Medicine, \\ Hangzhou 310003, P.R. China
}

Received October 12, 2011; Accepted December 23, 2011

DOI: $10.3892 / 01.2012 .557$

\begin{abstract}
CD14 is the pivotal molecule in the diagnosis and therapy of CD14-associated diseases, and is important in bacteremia. The HeLa cell line is regarded as immortal due to its prolific character. The HeLa cell line is derived from human cervical cancer cells and has been widely used in cancer research and gene transfection. In the present study, we established the expression plasmid pcDNA3.1(+)-CD14, and transfected it into the human cervical cancer cell line HeLa to establish a stable cell line (HeLa-CD14) expressing human CD14 antigen on the membrane. After the human CD14 gene was cloned and sequenced through RT-PCR and T-A cloning techniques, the eukaryotic expression vector pcDNA3.1(+)CD14 was constructed by cleaving with double restriction endonucleases and ligating with T4 ligase. HeLa cells were transfected with the pcDNA3.1(+)-CD14 recombinant plasmid using Superfect transfection reagent. The cells were selected using G418 and the expression of human CD14 on the transfectant was confirmed by RT-PCR and immunohistochemistry. The expression of CD14 mRNA was significantly different between the blank pcDNA3.1(+)-transfected cell group and the pcDNA3.1(+)-CD14-transfected cell group $(\mathrm{p}<0.01)$. The fluorescence was significantly stronger on the established stable cell line than on the transiently transfected HeLa cells, and no visible fluorescence was observed in blank pcDNA3.1(+)transfected cells. In this study, the human CD14 transfectant, stable cell line HeLa-CD14, was successfully established, which may be used to study CD14 and cervical cancer in vitro and in vivo.
\end{abstract}

\section{Introduction}

CD14, which is mainly expressed on normal and malignant monocytes and macrophages, or weakly expressed on normal

Correspondence to: Professor Yong-Min Tang, Division of Hematology-Oncology, The Children's Hospital of Zhejiang University School of Medicine, Zhugan Lane No. 57, Hangzhou 310003, P.R. China

E-mail: y_m_tang@zju.edu.cn

Key words: CD14, transfectant, HeLa granulocytes, is not expressed on other cells, including T, B, natural killer (NK) and red blood cells, platelets, hematopoietic stem/progenitor and non-hematopoietic cells (1), and monocytes and macrophages are capable of internalizing immunocompounds when CD14 is recognized and combined with its antibody and ligands (2). The CD14 molecule may enhance the microbicidal ability of monocytes and macrophages through binding with Toll-like receptor 2 and 4 (TLR2 and 4). At present, the majority of laboratories continue to obtain their CD14-positive cells through VitD3 induction of U937 (3). This method neither guarantees the uniformity of CD14 expression nor allows convenient research on the CD14 mechanism in vivo. A previous study showed that there was a correlation between CD14 (monocyte/macrophage), EGF-R and HPV DNA in HPV-infected benign epithelium and dysplastic lesions of the uterine cervix (4). In order to study CD14 and its correlation with cervical cancer conveniently, we successfully established a cell line, HeLa-CD14, which stably expressed human CD14 on its membrane according to the high transfection efficiency of the human cervical cancer cell line HeLa.

\section{Materials and methods}

Materials. Mononuclear cells were separated from human peripheral blood cells. 2F9 murine monoclonal antibody was preserved in our laboratory. High Fidelity Platinum ${ }^{\circledR}$ Taq, E. coli DH5 $\alpha$, Taq DNA polymerase, Olig(dT)12-18 primer, M-MLV reverse transcriptase, RNasin ${ }^{\circledR}$, RQ1 RNAasefree DNAase, pGEM $^{\circledR}$-T easy Vector system, X-gal, IPTG, pcDNA3.1(+) plasmid, Dulbecco's modified Eagle's medium (DMEM) and TRIzol total RNA extract reagent were purchased from Invitrogen. DNase-free RNase was obtained from Roche. Restricted incision enzymes EcoRI and XbaI, G418, T4 DNA ligase were purchased from Gibco BRL. QIAquick Gel Extraction kit and Superfect transfection reagent were obtained from Qiagen. Fluorescein isothiocyanate (FITC) labeling goat anti-mouse $\operatorname{IgG}(\mathrm{H}+\mathrm{L})$ was from Peking Zhongshang Biotechnological Limited Corporation, and primers were synthesized by Shanghai Biotech Limited Company. SYBR ${ }^{\circledR}$ Green PCR Master Mix was from ABI. The human cervical cancer cell line HeLa was kindly provided by the Cancer Research Center of Zhejiang University.

The study was approved by the ethics committee of the Children's Hospital of Zhejiang University School of Medicine. 
Cell culture. HeLa cells were maintained in DMEM supplemented with $10 \%(\mathrm{v} / \mathrm{v})$ fetal bovine serum, glutamine $2 \mathrm{mmol} / \mathrm{l}$, penicillin $(100 \mathrm{IU} / \mathrm{ml})$ and streptomycin $(100 \mathrm{IU} / \mathrm{ml})$. The selection-medium was made by adding G418 sulfate (500 $\mu \mathrm{g} /$ $\mathrm{ml}$ ) into complete medium. Suspended cells were obtained with digestion solution containing $0.25 \%$ trypsin plus $0.02 \%$ EDTA.

Construction of the pcDNA3.1(+)-CD14 plasmid. For total RNA isolation from the human mononuclear cells separated from peripheral blood cells, TRIzol reagent was used according to the manufacturer's instructions. The cDNA of CD14 was synthesized from the total RNA template using murine leukemia virus reverse transcriptase and Olig(dT)12-18 primers. The CD14 gene was amplified with the High Fidelity Platinum Taq polymerase and the primers used are listed in Table I.

Conditions for PCR amplification were described as follows: 30 cycles of denaturation at $94^{\circ} \mathrm{C}$ for $30 \mathrm{sec}$, annealing at $57^{\circ} \mathrm{C}$ for $30 \mathrm{sec}$, and extension at $72^{\circ} \mathrm{C}$ for $50 \mathrm{sec}$, and a final $72^{\circ} \mathrm{C}$ extension for $7 \mathrm{~min}$. The final PCR products were cloned into a pGEM-T easy vector and recombinant plasmids were sequenced. The verified recombinant plasmids were treated with $E c o$ RI and $X b a I$, then the gene of interest was cloned into a pcDNA3.1(+) vector and the positive expression vector was named pcDNA3.1(+)-CD14. All DNA manipulation and bacterial transformation were based on the methods described by Sambrook and Russell (5).

Transient transfection and determination. HeLa cells were transfected with pcDNA3.1(+) and pcDNA3.1(+)-CD14, respectively, by the Superfect transfection reagent according to the manufacturer's instructions. Following 24 of transfection, the determination of CD14 expression was conducted from mRNA and protein aspects by quantitative PCR (qPCR) and immunohistochemistry, respectively. The primers of CD14 for qPCR were as follows: CD14-F, ACTTGCACTTTCCAGCTTGCG; CD14-R, CCTGTGGGCGTCTCCATTCC. Simultaneous detection of the GAPDH gene was conducted as an internal control. The primers of GAPDH were listed as follows: GAPDH-F, 5'-AGA AGGCTGGGGCTCATTTG-3'; GAPDH-R, 5'-AGGGGCCATCCACAGTCTTC-3'. The relative variation rate of the CD14 gene was calculated according to the ${ }^{\Delta \Delta} \mathrm{Ct}$ method.

Stable transfection and characterization. HeLa cells were transfected with pcDNA3.1(+) and pcDNA3.1(+)-CD14 by the Superfect transfection reagent according to the stable transfection procedures for the adherent cells. The transfected HeLa cells were cultured with selection-medium (G418 concentration $500 \mu \mathrm{g} / \mathrm{ml}$ ) from the second day of transfection. Following characterization by RT, qPCR and immunohistochemistry, the selected positive clonal cells were cultured under the G418 selection-medium for approximately 2 months, then the HeLa-CD14 cells were maintained in the culture medium containing $300 \mu \mathrm{g} / \mathrm{ml} \mathrm{G} 418$. The cell line transfected with pcDNA3.1(+)-CD14 was designated HeLa-CD14.

Statistical analysis. The relative variation rate of the CD14 gene was statistically analyzed using the t-test by SPS 11.0 computer software.
Table I. Primers for cloning of the CD14 gene.

$\begin{array}{ll}\text { Forward: } & \text { 5'-GAATTCCTTATCGACCATGGAGCGCGCGTCC-3' } \\ & (\text { EcoRI }) \\ \text { Reverse: } & \text { 5'-TCTAGATTAGGCAAAGCCCCGGGC-3' } \\ & (\mathrm{Xba \textrm {I } )}\end{array}$

The restriction enzymes are shown in parentheses and their sites are underlined.

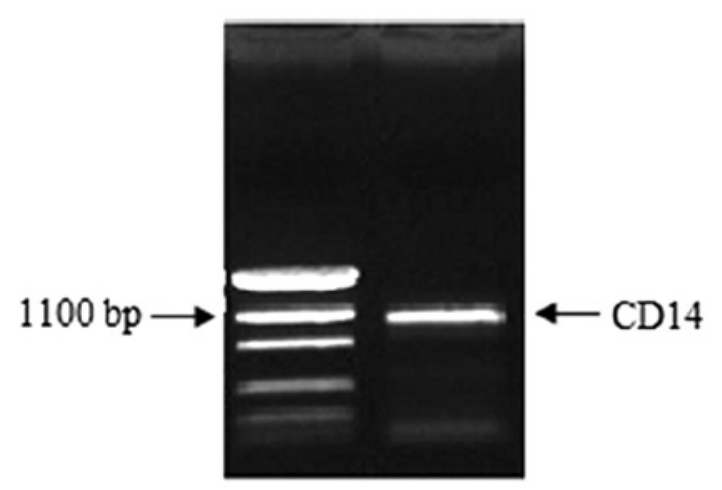

Figure 1. PCR product of the CD14 gene. The CD14 gene amplified by PCR with 1128-bp size was detected by $0.8 \%$ agarose gel electrophoresis. PCR, polymerase chain reaction.

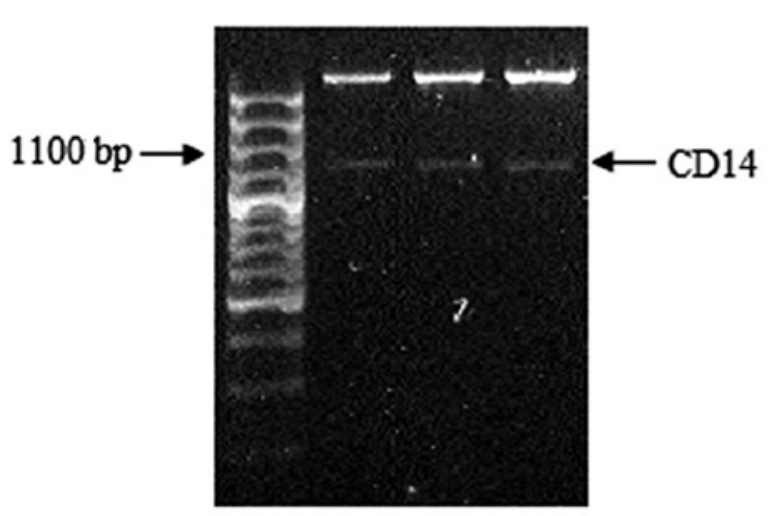

Figure 2. Cleaving of pcDNA3.1(+)-CD14 with EcoRI and XbaI. The CD14 gene with 1128-bp size was observed in three individual positive recombined plasmids.

\section{Results}

Construction of the pcDNA3.1(+)-CD14 plasmid. The band with 1128-bp size was detected through $0.8 \%$ agarose gel electrophoresis (Fig. 1). The result from the sequencing of pGEM-T Easy-CD14 and GeneBank data revealed that the cloned CD14 gene sequence was correct. After cleaving with EcoRI and $X b a I$ enzymes, the 1128-bp sized band was also detected on the agarose gel electrophoresis from three positive recombinant plasmids (Fig. 2).

Dissociation curves of CD14 and GAPDH primers. According to the dissociation curves of CD14 and GAPDH primers, each 


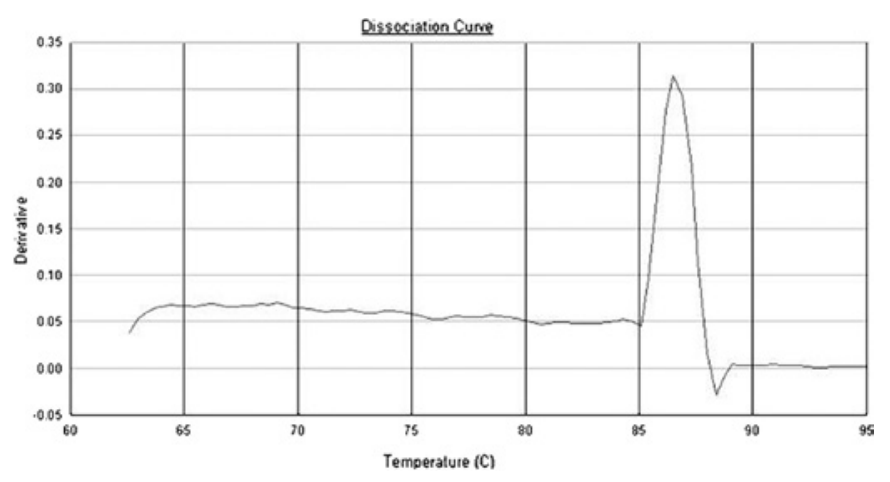

Figure 3. The dissociation curve of CD14 primers.

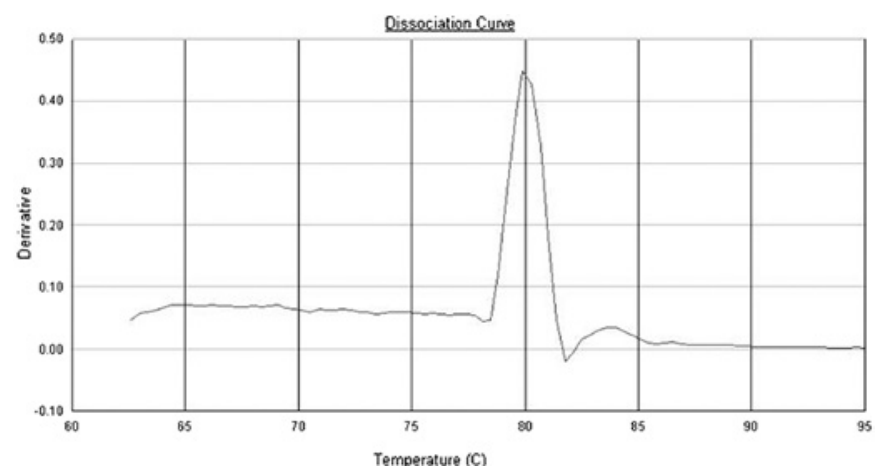

Figure 4. The dissociation curve of GAPDH primers.

pair of primers showed only a single peak at approximately 86 and $80^{\circ} \mathrm{C}$, respectively (Figs. 3 and 4).

Transient transfection and determination. The expression of CD14 mRNA was significantly increased $(\mathrm{p}<0.01)$ in the HeLa cells after being transiently transfected with pcDNA3.1(+)-CD14 (Fig. 5A). The CD14 protein could also be observed by immunohistochemistry with strong green fluorescence on the cellular membrane (Fig. 6A and B).

Stable transfection and characterization. After stable transfection and drug selection for approximately 2 months, the expression of human CD14 mRNA significantly increased $(\mathrm{p}<0.01)$ in the HeLa-CD14 cells compared to the empty vectortransfected HeLa cells through RT-PCR detection (Fig. 5B). The CD14 molecules were also visible on the membrane by immunohistochemistry with stronger green fluorescence than the transiently transfected cells (Fig. 6C and D).

\section{Discussion}

CD14 takes its name from its inclusion in the cluster of the differentiation group of cell surface marker proteins. It is expressed mainly by macrophages, dendritic cells and monocytes, and weakly (at 10 times lesser extent) by neutrophil granulocytes. CD14 exists in two forms: the membrane form (mCD14), which is anchored into the membrane by a glycosylphosphatidylinositol tail, and the soluble form (sCD14), which appears in plasma. sCD14 either appears after shedding of
A

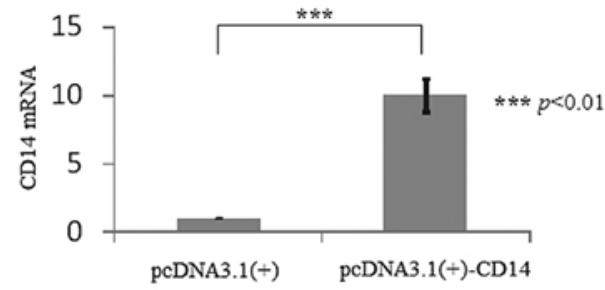

B

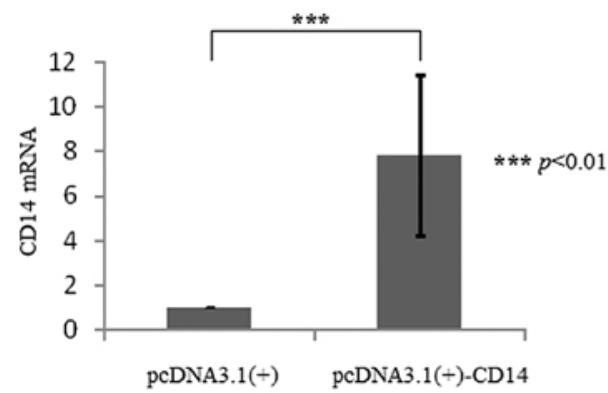

Figure 5. The CD14 mRNA expression between HeLa cells transfected with pcDNA3.1(+) and pcDNA3.1(+)-CD14. (A) Transient transfection; $p<0.01$ between the pcDNA3.1(+) and pcDNA3.1(+)-CD14 groups. (B) Stable transfection; $\mathrm{p}<0.01$ between the pcDNA3.1(+) and pcDNA3.1(+)-CD14 groups.
A

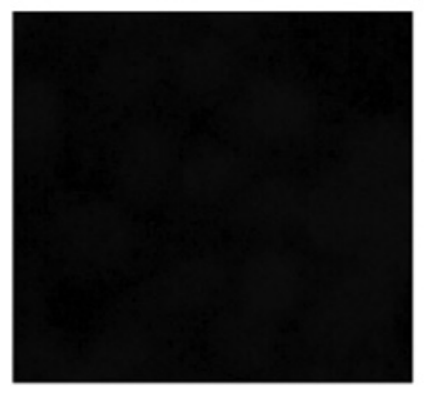

C

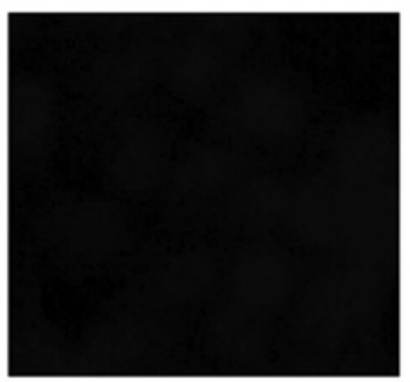

B

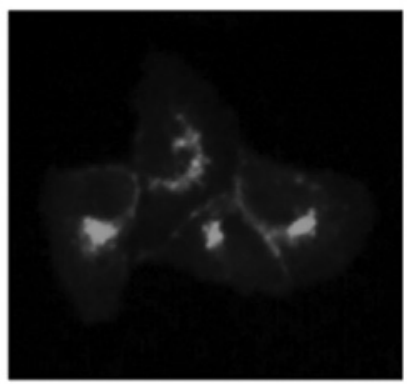

D

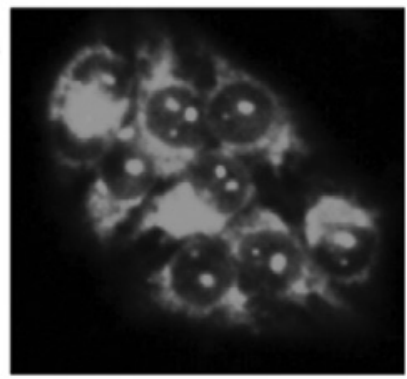

Figure 6. The immunohistochemistry of HeLa cells transfected with pcDNA3.1(+) and pcDNA3.1(+)-CD14. (A) Transiently transfected with pcDNA3.1(+). (B) Transiently transfected with pcDNA3.1(+)-CD14. (C) Stably transfected with pcDNA3.1(+). (D) Stably transfected with pcDNA3.1(+)-CD14.

mCD14 (48 kDa) or is directly secreted from intracellular vesicles $(56 \mathrm{kDa})(6)$.

During sepsis, it serves as a co-receptor (along with the TLR 4 and MD-2) for the detection of, and combination with, bacterial lipopolysaccharide (LPS) $(7,8)$. CD14 may bind to LPS only in the presence of lipopolysaccharide-binding protein (LBP). Although LPS is considered as its main ligand, CD14 also recognizes other pathogen-associated molecular patterns (PAMPs). Studies have shown that dibutyryl cyclic adenosine 
3',5'-monophosphate (dB-cAMP) promoted the differentiation of argyrophil small cell carcinoma cells (ASCC) of the uterine cervix to macrophages, with two specific antigenic markers, CD14 and human leukocyte antigen-DR (9). M2 macrophages are transformed to activated M1 macrophages by $\mathrm{CD} 4^{+} \mathrm{Th} 1$ cells under the influence of prostaglandin E2 and IL-6 in cervical carcinoma (10). This may indicate that bacterial infection or the CD14 molecule plays a role in cervical cancer. CD14 may also induce endocytosis through interaction between antibody and antigen, which provides a great advantage to antibody-targeting therapy for CD14-associated disease. In order to facilitate studies using CD14 models in vitro and in vivo, we established the stable and highly efficient cell line HeLa-CD14 using the Superfect transfection reagent.

The human CD14 gene is localized at 5q31.1 with 1338 nucleotides of genomic DNA, encoding a protein with 375 amino acid residues from 76 to 1200 nucleotides (11). The genome is composed of two exons, one of which only has a start codon ATG next to an intron of 88 nucleotides (12). To avoid the contamination of genomic DNA, we used RNAase-free DNAase to digest residual DNA, so as to guarantee the template of PCR derived from cDNA other than CD14 genomic DNA. Considering that the size of the CD14 gene is more than $1 \mathrm{~kb}$ and the mismatch occurrence when using regular DNA polymerase, we conducted the PCR with high fidelity Taq polymerase purchased from Invitrogen. The blast analysis from GeneBank showed that the cloned CD14 gene of 1128 bp was exactly the same.

HeLa (also Hela or hela cells) is known to be a cervical carcinoma cell line frequently used in tumor research. This cell line has revealed much efficiency and stability in extrinsic gene transfection (13-15); in this study, we selected the cell line as the host cell and conducted the human CD14 gene transfection into it.

G418 (also known as geneticin) is an aminoglycoside antibiotic, which is mostly used in laboratory research to select genetically engineered cells. Commonly, for mammalian cells, a concentration of approximately $400 \mu \mathrm{g} / \mathrm{ml}$ is used for selection and $200 \mu \mathrm{g} / 1$ for maintenance (16). The cells were resistant to G418 following transfection with pcDNA3.1(+) and pcDNA3.1(+)-CD14, which have the neo gene. After almost 2 months of selection with $500 \mu \mathrm{g} / \mathrm{ml} \mathrm{G} 418$, we obtained a stable cell line HeLa-CD14, which intensively expressed human CD14 on the membrane. From the results of qPCR, the relative variation rate of the CD14 gene between pcDNA3.1(+) and pcDNA3.1(+)-CD14-transfected cells was significant ( $<<0.01$ ), both on transient and stable transfection (Fig. 5A and $\mathrm{B}$ ), and the fluorescence of the stable HeLa-CD14 cell line was stronger than that in the transiently transfected cells (Fig. 6B and D).

In conclusion, we successfully established a human CD14-positive cell line, HeLa-CD14, and the protein was expressed stably and strongly on the cellular membrane. This study constructed a research cell line to investigate human CD14 molecules and the relationship between CD14 and cervical cancer, not only in vitro but also in animal models.

\section{Acknowledgements}

This study was sponsored by the National Natural Science Foundation of China (project no. 30901327).

\section{References}

1. Mason D, Simmons D, Buckley C, et al: Leukocyte Typing VII. Oxford University Press Inc, New York, 2002.

2. Poussin C, Foti M, Carpentier JL and Pugin J: CD14-dependent endotoxin internalization via a macropinocytic pathway. J Biol Chem 273: 20285-20291, 1998

3. Botling J, Oberg F, Torma H, Tuohimaa P, Blauer M and Nilsson K: Vitamin D3- and retinoic acid-induced monocytic differentiation: interactions between the endogenous vitamin D3 receptor, retinoic acid receptors, and retinoid $\mathrm{X}$ receptors in U-937 cells. Cell Growth Differ 7: 1239-1249, 1996.

4. Suzuki M, Furugen Y, Miyazaki R, Yamamoto T and Takada M: Sequential changes of HPV DNA, the expression of EGF-R and CD-14, and histopathological findings in HPV infected benign epithelium and dysplastic lesions of the uterine cervix. Nihon Sanka Fujinka Gakkai Zasshi 44: 805-812, 1992 (In Japanese).

5. Sambrook J and Russell DW: Molecular Cloning: A Laboratory Manual, 3rd edition. Cold Spring Harbor Laboratory Press, New York, 2001

6. Kirkland TN and Viriyakosol S: Structure-function analysis of soluble and membrane-bound CD14. Prog Clin Biol Res 397: 79-87, 1998.

7. Triantafilou M, Sawyer D, Nor A, Vakakis E and Triantafilou K: Cell surface molecular chaperones as endogenous modulators of the innate immune response. Novartis Found Symp 291: 74-79; discussion 79-85, 137-140, 2008.

8. Dziarski R, Viriyakosol S, Kirkland TN and Gupta D: Soluble CD14 enhances membrane CD14-mediated responses to peptidoglycan: structural requirements differ from those for responses to lipopolysaccharide. Infect Immun 68: 5254-5260, 2000.

9. Ichimura H, Sakashita N, Iida T, et al: Differentiation of a cell line of human cervical argyrophil small cell carcinoma to macrophage lineage cells. Jpn J Cancer Res 90: 523-529, 1999.

10. Heusinkveld M, de Vos van Steenwijk PJ, Goedemans R, et al: M2 macrophages induced by prostaglandin E2 and IL-6 from cervical carcinoma are switched to activated M1 macrophages by CD4 ${ }^{+}$Th1 cells. J Immunol 187: 1157-1165, 2011.

11. Baldini M, Lohman IC, Halonen M, Erickson RP, Holt PG and Martinez FD: A polymorphism* in the 5' flanking region of the CD14 gene is associated with circulating soluble CD14 levels and with total serum immunoglobulin E. Am J Respir Cell Mol Biol 20: 976-983, 1999.

12. Ferrero E, Hsieh CL, Francke U and Goyert SM: CD14 is a member of the family of leucine-rich proteins and is encoded by a gene syntenic with multiple receptor genes. J Immunol 145: 331-336, 1990.

13. Capes-Davis A, Theodosopoulos G, Atkin I, et al: Check your cultures! A list of cross-contaminated or misidentified cell lines. Int J Cancer 127: 1-8, 2010.

14. Rahbari R, Sheahan T, Modes V, Collier P, Macfarlane C and Badge RM: A novel L1 retrotransposon marker for HeLa cell line identification. Biotechniques 46: 277-284, 2009.

15. Masters JR: HeLa cells 50 years on: the good, the bad and the ugly. Nat Rev Cancer 2: 315-319, 2002.

16. Yoon YG and Koob MD: Selection by drug resistance proteins located in the mitochondria of mammalian cells. Mitochondrion 8: 345-351, 2008. 